\title{
La Respuesta Educativa al Alumnado con Trastorno del Espectro del Autismo (TEA) en España: Un avance Desigual
}

\author{
The Educational Response to Students with Autism Spectrum \\ Disorder (ASD) in Spain: An Unequal Advance
}

\author{
Belén de la Torre González * \\ Elena Martín
}

Universidad Autónoma de Madrid, España

\begin{abstract}
En los últimos años, la respuesta educativa al alumnado con TEA ha avanzado hacia alternativas más inclusivas en España. Este hecho representa un claro compromiso de las administraciones por acercar a este colectivo de alumnado a los centros ordinarios combinando así una atención intensiva y especializada con los aprendizajes en sus aulas de referencia junto a sus iguales. Sin embargo, a pesar de esta buena noticia para nuestro sistema, las diferencias en las propuestas que han impulsado las distintas Comunidades Autónomas (CCAA) ponen de manifiesto un riesgo de desigualdad de oportunidades para el alumnado con TEA y de falta de equidad en el sistema educativo español. En este artículo, a través del análisis de la normativa existente en las distintas CCAA, se expondrán las principales diferencias encontradas en la presencia del alumnado con TEA en los centros ordinarios. Esta situación genera importantes desafíos para nuestro sistema educativo. En un sistema descentralizado, el equilibrio entre la distribución de competencias en materia educativa entre la Administración central y las distintas autonomías, y la garantía de igualdad de oportunidades para todo el alumnado debería ser una prioridad para un sistema educativo que quiere avanzar con determinación hacia la inclusión educativa.
\end{abstract}

Descriptores: Inclusión; TEA; Sistema educativo; Políticas inclusivas; Equidad.

In recent years, the educational response to students with ASD has progressed towards more inclusive alternatives in Spain. This fact represents a clear commitment of the administrations to bring this group of students closer to the ordinary centers combining intensive and specialized attention with the learning in their reference classrooms with their peers. However, despite this good news for our system, the differences in the proposals promoted by the different Autonomous Communities (Autonomous Communities) are an example of inequality of opportunities for students with ASD and lack of equity in the Spanish education system. In this article, through the analysis of the existing regulations in the different Autonomous Communities will be presented the main differences found in the presence of students with ASD in the ordinary centers. This situation generates important challenges for our education system. In a decentralized system, the balance between the distribution of competences in educational matters between the Central Administration and the different autonomies, and the guarantee of equal opportunities for all students should be a priority for a system education that wants to move forward with determination towards educational inclusion.

Keywords: Inclusion; ASD; Education system; Inclusive policies; Equity.

*Contacto: belendelatorre22@hotmail.com

ISSN: 2254-3139

www.rinace.net/riejs/

revistas.uam.es/riejs
Recibido: $\quad 12$ de septiembre 2019

$1^{\text {a }}$ Evaluación: 1 de diciembre 2019

$2^{\text {a }}$ Evaluación: 14 de febrero 2020

Aceptado: $\quad 25$ de marzo 2020 


\section{Introducción ${ }^{1}$}

Avanzar hacia un sistema educativo inclusivo supone un gran reto para cualquier país, y España no es una excepción. Después de años de grandes avances en la década de los noventa, como consecuencia de la descentralización de las competencias, la inestabilidad política y la falta de consenso en materia educativa, se ha producido un estancamiento significativo.

A pesar de esta disminución de una respuesta de inclusión plena para los estudiantes con mayores dificultades, en el caso del alumnado con TEA, en los últimos años han proliferado distintas fórmulas por parte de las Comunidades Autónomas siendo el colectivo que dispone de mayores propuestas inclusivas a lo largo de la geografía española. Este artículo analiza las diferentes políticas educativas que las diferentes CCAA han adoptado para la atención de este alumnado como un indicador de avance en el proceso de inclusión educativa pero también como un ejemplo de desigualdad territorial al constatar diferencias notables entre las iniciativas que se desarrollan en cada CCAA.

\section{El panorama internacional y nacional}

La educación siempre ha sido una preocupación de todos los gobiernos y Organismos Internacionales encargados de velar por el derecho a la misma incluyendo el de las personas con discapacidades (Martínez, De Haro y Escarbajal, 2010).

Un claro ejemplo de ello son las aportaciones de los distintos Organismos Internacionales, Foros y Declaraciones donde se reconoce el derecho a la Educación para Todos y a la Educación Inclusiva. Entre las más relevantes en el tema que nos ocupa, cabe señalar la Declaración Universal de los Derechos Humanos que, proclamada por la Asamblea General de las Naciones Unidas en 1948, marcó un primer hito al establecer por primera vez el derecho a la educación de todas las personas.

Por su parte, la Conferencia Mundial sobre Necesidades Educativas Especiales, organizada por el Gobierno español en cooperación con la UNESCO en 1994, supuso un avance significativo a nivel internacional en materia de atención al alumnado con necesidades educativas especiales y, en palabras de Lena Saleh (2004), situó a España en los años 90 en el centro del debate y la innovación educativa en esta temática.

En tercer lugar, la Convención Internacional sobre los Derechos de las Personas con Discapacidad se constituyó en 2006 como un instrumento internacional de las Naciones Unidas a favor de los derechos humanos destinado a proteger los derechos y la dignidad de las personas con discapacidad. Esta Convención fue ratificada por España en 2008. En ella se establece en el artículo 24 que los Estados Partes deben asegurar en materia de educación que:

- Las personas con discapacidad no queden excluidas del sistema general de educación por motivos de discapacidad, y que los niños y las niñas con

\footnotetext{
${ }^{1}$ En el artículo se aborda la respuesta educativa del alumnado con Trastorno del espectro del autismo (TEA) también denominado alumnado con Trastornos Generalizados del Desarrollo (TGD) por algunas CCAA. No obstante, se ha optado por el uso de la terminología alumnado con TEA siguiendo la clasificación vigente del DSM-V.
} 
discapacidad no queden excluidos de la enseñanza primaria gratuita y obligatoria ni de la enseñanza secundaria por motivos de discapacidad;

- Las personas con discapacidad puedan acceder a una educación primaria y secundaria inclusiva, de calidad y gratuita, en igualdad de condiciones con las demás, en la comunidad en que vivan;

- Se hagan ajustes razonables en función de las necesidades individuales;

- Se preste el apoyo necesario a las personas con discapacidad, en el marco del sistema general de educación, para facilitar su formación efectiva;

- Se faciliten medidas de apoyo personalizadas y efectivas en entornos que fomenten al máximo el desarrollo académico y social, de conformidad con el objetivo de la plena inclusión.

Por último, los Objetivos de Desarrollo del Milenio (ODM) y los Objetivos de Desarrollo Sostenible (ODS) de Naciones Unidas, que en la actualidad se concretan en la Agenda 2030, también recogen, entre sus 17 objetivos de aplicación universal para lograr un mundo sostenible en el año 2030, uno destinado a la educación (objetivo 4) centrado en garantizar una educación inclusiva, equitativa y de calidad y promover oportunidades de aprendizaje durante toda la vida para todos.

A nivel nacional, el sistema educativo español, al igual que otros países, ha hecho un esfuerzo progresivo través de su legislación por adaptar sus planteamientos educativos relacionados con la atención a las personas con discapacidad tanto a las tendencias teóricas internacionales como a las referencias internacionales anteriormente citadas.

Vislie (1995) señala que los países occidentales han optado por dos tipos de estrategias en la integración del alumnado con discapacidad. Por una parte, se encuentran países que han puesto su foco de atención en la reforma del sistema de educación especial. Y por otra, países cuya prioridad ha sido la transformación de la escuela ordinaria entendiendo esta alternativa como la estrategia más efectiva para acoger al alumnado con mayores dificultades. El caso de España se puede valorar a través de los diferentes textos legales aprobados de forma progresiva que se partió de la primera estrategia para posteriormente ir transitando a la segunda (Marchesi, 2002).

Las aportaciones más significativas en materia educativa se desarrollaron a finales de la década de los años 80 y durante toda la década de los años 90, períodos en los que la aprobación de, entre otras normas, la Ley 13/1982, de 7 de abril, de Integración Social de los Minusválidos (LISMI) y el Real Decreto 334/1985, de 6 de marzo, de Ordenación de la Educación Especial establecerían los preámbulos de una nueva Ley educativa, la Ley Orgánica 1/1990, de 3 de octubre, de Ordenación General del Sistema Educativo (LOGSE) apostaron con determinación y compromiso político hacia un único sistema educativo comprensivo en el que la equidad constituyese una de sus señas de identidad.

A partir de ese momento, a través de su desarrollo normativo (Real Decreto 696/1995, de 28 de abril, de ordenación de la educación de los alumnos con necesidades educativas especiales y el Real Decreto 299/1996, de 28 de febrero, de ordenación de las acciones dirigidas a la compensación de desigualdades en educación, entre otros) se produjeron serios avances en la ordenación y atención del alumnado con necesidades educativas especiales y aquel que se encontraba en situación de desventaja educativa por cuestiones sociales o culturales. 
A pesar de estos avances sustanciales, hay que esperar hasta el año 2006, en el que se aprueba la Ley Orgánica de Educación, 2/2006, de 3 de mayo (LOE) para encontrar por primera vez una alusión al concepto de inclusión educativa en su Preámbulo y principios reguladores. Sin embargo, la aprobación de esta nueva ley educativa no ha generado ningún cambio conceptual y la política educativa continúa siendo la misma.

Posteriormente, aunque la Ley Orgánica 8/2013, de 9 de diciembre, para la Mejora de la Calidad Educativa (LOMCE) continúa manteniendo el principio de inclusión educativa, sus principios centrados en las evaluaciones individuales, la flexibilización de las trayectorias educativas, la racionalización en la oferta, así como su interés en la mejora de los resultados educativos y en los alumnos excelentes rompen con el paradigma comprensivo de la educación que hasta el momento se habían conseguido a través de los distintos textos legislativos anteriormente comentados.

A esta situación hay que añadir la ausencia de normativa de carácter básico en los últimos veinte años que promueva la implementación de una política educativa que haga efectiva una educación inclusiva a lo largo de la geografía española. Esta circunstancia ha supuesto que cada CCAA, como se desarrolla más adelante, haya avanzado a diferentes ritmos y con planteamientos educativos en gran medida distintos. Así lo reflejan algunos estudios que han puesto de manifiesto la existencia de diferencias notables en los procesos de integración e inclusión educativa en función de la etapa escolar, las necesidades educativas del alumnado y CCAA. Por citar únicamente dos de los que consideramos más relevantes, el trabajo de Sandoval, Simón y Echeita (2012) centrado en el análisis y la valoración crítica de las funciones del profesorado de apoyo destaca entre sus conclusiones las grandes desigualdades existentes dentro del sistema educativo español en la atención al alumnado con mayores dificultades, así como la falta de consenso y de debate en relación a la estructura y las dinámicas de trabajo del profesorado de apoyo. Estas conclusiones se basan en los resultados encontrados en relación a una excesiva vinculación del trabajo directo del profesorado de apoyo con el alumnado con mayores dificultades, así como en las diferencias de ratio entre profesorado especializado y alumnado en las normativas de las distintas CCAA.

Por su parte, recientemente Verdugo y otros (2018) han impulsado un trabajo minucioso en relación al análisis de la adaptación de la normativa española y autonómica en materia de inclusión educativa y social a la Convención de las Naciones Unidas sobre los derechos de las personas con Discapacidad. Entre sus múltiples conclusiones, al igual que en el trabajo anterior, se destaca la gran variabilidad en cuanto al grado de concreción y desarrollo entre las distintas CCAA, lo que los autores interpretan como consecuencia, entre otros factores, de un marco estatal legislativo poco preciso.

Centrando el análisis en la respuesta educativa del alumnado con TEA, cabe señalar que su planteamiento ha pasado por numerosos cambios conceptuales. Hay que tener en cuenta que es un trastorno relativamente reciente, recordemos que Leo Kanner (1943) habla por primera vez de "los trastornos autistas del contacto afectivo".

Además, en su estudio podemos distinguir tres épocas en las que la comprensión del trastorno, y, por tanto, la intervención que se desarrollaba se basó en función de las distintas concepciones. Por otra parte, los cambios en los manuales diagnósticos han generado grandes controversias y cierto desconcierto en distintos sectores. Y, por último, entre otros elementos, la propia heterogeneidad tanto en la presentación del trastorno 
como en su evolución ha conllevado que el tipo de intervención con este alumnado haya sido muy cambiante a lo largo del tiempo.

En la actualidad, podríamos identificar cinco dilemas principales en la atención del alumnado con TEA: qué características deben reunir los centros para garantizar una óptima escolarización, cómo equilibrar los aprendizajes del currículo general y el currículo específico que estos estudiantes requieren, qué estrategias metodológicas son las más eficaces para su enseñanza, cómo evaluar la evolución y los progresos educativos, y por último, cómo incorporar la respuesta a estas preguntas en la organización y funcionamiento de un centro educativo ordinario.

Para los resultados que a continuación se van a presentar, es pertinente centrarse en el primer y último dilema identificado. El primer dilema presentado se relaciona con las características que deben reunir los centros educativos para poder atender de forma ajustada al alumnado con TEA. Rivière (2001) ya identificaba que los centros específicos de educación especial para niños autistas únicamente estaban justificados si cumplían tres condiciones: un claustro compuesto de expertos en autismo y en trastornos generalizados del desarrollo (TGD) con altos niveles de destrezas en la educación y tratamiento de niños con autismo; centros cuyas ratios alumno/ docente se ubicasen entre tres y cinco alumnos/as; así como la disponibilidad de especialistas en audición y lenguaje, psicomotricidad o fisioterapia, y personal complementario de apoyo.

Palomo (2017) aporta la revisión de dos investigaciones posteriores de gran relevancia en este tema. La primera de ella es la desarrollada por Strain y Bovet (2011) en la que identificaron que los alumnos con TEA que pudieron beneficiarse de un modelo de escolarización inclusivo con sólidos apoyos educativos y una planificación rigurosa en la respuesta educativa mostraron importantes avances en el desarrollo cognitivo, en el lenguaje y en las habilidades sociales, así como una reducción y en la sintomatología TEA y en los problemas de conducta. Por su parte, Martins, Harris y Handleman (2014) también apoyan la pertinencia de una educación inclusiva encontrando evidencia a favor de que la escolarización ordinaria para el alumnado con TEA en las distintas etapas es la más mejor opción siempre que se cuenten con los apoyos educativos y se planifique de forma rigurosa la respuesta educativa, constituyendo estos dos factores, elementos imprescindibles.

En el caso del último dilema relacionado con cómo incorporar las respuestas a las preguntas anteriormente señaladas en el marco de la organización y funcionamiento de un centro educativo, en la literatura científica se pueden encontrar sólidas aportaciones que arrojan información muy valiosa. Rivière (2001) señalaba la pertinencia de centros escolares pequeños en tamaño y número de alumnos; estructurados y con estilos didácticos directivos y formas de organización que faciliten la anticipación de la jornada escolar; comprometidos con la atención a la diversidad y en especial con el trabajo con el alumnado con TEA; con disponibilidad de recursos complementarios, en especial del perfil especialista en psicopedagogía con funciones de orientación y de logopedia; y por último, centros en los que se proporcione a los iguales claves para comprender y apoyar los aprendizajes y relaciones de sus compañeros/as con TEA. Por su parte, Bergerson y otros (2003) señalaban que cualquier programa educativo de buena calidad en la atención al alumnado con TEA debe contemplar la participación de la familia, la evaluación completa de destrezas y dificultades, el desarrollo de un plan para definir claramente las metas y objetivos de trabajo, la incorporación de estrategias de enseñanza eficaces, la evaluación 
de la intervención, una alta estructuración del entorno, la aplicación de la evaluación funcional de la conducta problemática ( en caso de existir), el cuidado en las transiciones educativas, el trabajo con los iguales, y un enfoque de equipo de trabajo exhaustivo que permita organización una respuesta educativa sólida.

Por último, la investigación impulsada por Charman y otros (2011) fue capaz de traducir los resultados encontrados en 16 escuelas que atendían a alumnos con edades comprendidas entre los 2 y 19 años en quince principios de "buenas práctica" en la educación para personas con autismo.

Entre estos quince principios se encuentran entre otros, las altas expectativas hacia el progreso del alumnado, el trabajo conjunto y cercano con las familias, la implementación de estrategias metodológicas innovadoras y métodos basados en la evidencia, la participación activa del alumnado en la vida del centro y en sus propios aprendizajes, el seguimiento sistemático de la evolución del alumnado a través de distintos métodos y estrategias, el trabajo articulado entre distintos profesionales con altos niveles de cualificación procedentes de ámbitos diferentes, y un liderazgo educativo consolidado para poder implementar todos estos principios en los centros educativos.

La evidencia acumulada en estos estudios ha ido calando en las administraciones educativas y ha dado lugar a que, de forma progresiva, la escolarización del alumnado con TEA haya sufrido notables cambios apostando por una escolarización en contextos educativos ordinarios en detrimento de los centros específicos.

Este artículo pretende poner de manifiesto esta cuestión a través de un análisis minucioso de las políticas públicas de atención al alumnado con TEA en las distintas CCAA. Para ello, de forma comparada se expondrán los puntos comunes identificados, pero también los aspectos diferenciadores que generan indicadores de inequidad y desigualdad de oportunidades a lo largo de la geografía española.

\section{Método}

La investigación desarrollada cuyos resultados parciales se exponen a continuación en el próximo apartado se ha desarrollado en el marco de la tesis doctoral: "La inclusión del alumnado con Trastorno del espectro del autismo (TEA) en Educación Infantil: análisis de buenas prácticas en la Comunidad de Madrid”. Un trabajo en el que, a través de una metodología de estudio de casos múltiples, se han identificado y analizado los aspectos clave para el desarrollo de prácticas inclusivas para el alumnado con TEA en la Educación Infantil a partir del modelo de escolarización preferente para alumnado con TEA que impulsa la Comunidad de Madrid.

En este contexto y conscientes de la necesidad de conocer con detalle la realidad española para contextualizar el estudio empírico, se ha realizado un trabajo exhaustivo de análisis comparado de las distintas políticas públicas desarrolladas por las diferentes administraciones autonómicas en la atención a este colectivo.

Para ello, en primer lugar, se ha identificado, a través de las páginas web de las Consejerías de Educación, la normativa vigente (hasta el curso escolar 2018/2019) que hacía alusión a la respuesta educativa del alumnado con TEA. A este respecto cabe señalar tres cuestiones a tener en cuenta en la información que a continuación se presenta. 
Por una parte, se ha analizado la normativa disponible en la que se hacía una referencia explícita al alumnado con TEA a través de las páginas webs de las Consejerías de Educación. Esto significa que puede que algunas CCAA dispongan de una normativa de mayor rango que también afecte al alumnado con TEA y no haya sido analizada al no ser específicamente destinada para organizar su respuesta educativa, así como que existan algunas iniciativas que, al no estar disponibles en los respectivos portales educativos, no hayan sido objeto de este análisis.

Por otra parte, se ha encontrado que algunas CCAA disponían de manuales de apoyo a su propuesta. Sin embargo, ni todas las CCAA contaban con este tipo de material de apoyo, ni en todas las CCAA que disponían de estos materiales su publicación se asociaba con la normativa desarrollada. Por estos motivos, y con la finalidad de mantener la misma tipología en cuanto a las fuentes de información analizadas, se ha optado por centrar el análisis únicamente en la normativa vigente.

Por último, es importante tener en cuenta que al tratarse de un análisis normativo algunos aspectos quedan abiertos a la interpretación del lector de la norma lo que supone cierta ambigüedad en la comprensión del texto, pero también en la aplicación de la misma por parte de los agentes educativos.

Una vez localizadas estas normas autonómicas, se han examinado con detalle para analizar el contenido de su legislación desde una perspectiva comparada. Este análisis comparativo se ha realizado teniendo en cuenta seis grandes dimensiones de las distintas propuestas: los aspectos formales, el encuadre conceptual, la vinculación de la iniciativa en el contexto de los centros educativos, el proceso de escolarización y las características de adscripción del alumnado, los recursos personales asociados y las indicaciones aportadas en relación a la organización de la respuesta educativa.

Finalmente, para cada una de las estas dimensiones, se han incorporado distintos descriptores que facilitan una visión global pero lo más específica posible de cómo se concreta la respuesta educativa al alumnado con TEA en cada CCAA.

En el cuadro 1 se recogen la totalidad de las dimensiones y descriptores analizados. No obstante, teniendo en cuentas las limitaciones en la extensión de este artículo, únicamente se expondrán los resultados asociados a las dimensiones vinculadas con los aspectos formales y el encuadre conceptual de cada una de las propuestas, ambas dimensiones asociadas, como se comentaba anteriormente, a una de las características definitorias del concepto de inclusión educativa: la presencia en entornos ordinarios del alumnado con mayores dificultades que, si bien debe ir asociada a la participación y el aprendizaje, es requisito sine qua non para la inclusión (Echeita, 2006).

\section{Resultados}

A continuación, se presentan los resultados preliminares del análisis comparado entre las políticas públicas de las CCAA en la atención al alumnado con TEA para las dos primeras dimensiones.

Respecto a la dimensión relacionada con el alcance de la propuesta de las distintas propuestas cabe señalar que la mayoría de las CCAA han optado por la denominación de "aulas" en la identificación de cada una de las propuestas. Excepcionalmente se ha establecido el nombre de "unidades" en el caso de la Cataluña, la Comunidad Valenciana y 
la Comunidad Foral de Navarra, y solamente en el caso de Aragón y la Comunidad de Madrid se han decantado por denominar sus propuestas como "centros" haciendo referencia a "centros de atención preferente a alumnos con TEA" o "centros de escolarización preferente para alumnado con TGD”, respectivamente.

Cuadro 1. Dimensiones y descriptores analizados a través de la revisión de la normativa vigente en las CCAA

\begin{tabular}{|c|c|}
\hline DIMENSIONES DE ANÁLISIS & DESCRIPTORES DE LA PROPUESTA \\
\hline \multirow{4}{*}{ Alcance de la propuesta } & Denominación \\
\hline & Año de puesta en funcionamiento \\
\hline & Titularidad de los centros en los que se oferta \\
\hline & Etapas educativas en las que se desarrolla \\
\hline \multirow{3}{*}{ Encuadre conceptual } & Modalidad educativa en la que se encuadra \\
\hline & Alumnado destinatario \\
\hline & Ratio \\
\hline \multirow{6}{*}{ Encuadre en el centro } & $\begin{array}{l}\text { Grado de decisión de la propuesta por parte del } \\
\text { centro }\end{array}$ \\
\hline & Relación con otros documentos/ medidas del centro \\
\hline & Formación del profesorado y vinculación con la \\
\hline & innovación educativa \\
\hline & Sensibilización de la comunidad \\
\hline & Seguimiento y evaluación \\
\hline \multirow{5}{*}{$\begin{array}{l}\text { Proceso de escolarización y } \\
\text { adscripción del alumnado }\end{array}$} & Perfil del alumnado destinatario \\
\hline & Intensidad de apoyos requeridos \\
\hline & Responsable de realizar la adscripción \\
\hline & Opinión de las familias o tutores legales \\
\hline & Revisión de la adscripción de la propuesta \\
\hline \multirow{4}{*}{ Recursos personales asociados } & Recursos personales adscritos \\
\hline & $\begin{array}{l}\text { Responsables de la coordinación y delimitación de } \\
\text { tiempos y espacios }\end{array}$ \\
\hline & Intervención del perfil de orientación \\
\hline & Apertura a los recursos externos \\
\hline \multirow{5}{*}{ Organización de la respuesta educativa } & Objetivos de trabajo y propuestas de intervención \\
\hline & $\begin{array}{l}\text { Indicaciones sobre la organización de la respuesta } \\
\text { educativa del alumnado }\end{array}$ \\
\hline & Indicaciones sobre la evaluación y evolución del \\
\hline & alumnado \\
\hline & $\begin{array}{l}\text { Indicadores sobre la elaboración de los informes de } \\
\text { seguimiento }\end{array}$ \\
\hline
\end{tabular}

Fuente: Elaboración propia.

Respecto al año de puesta en funcionamiento de las propuestas, la heterogeneidad es muy grande. Podemos identificar claramente al País Vasco como pionera de esta iniciativa en el año 1998, seguidos de Andalucía en el 2002 y la Comunidad de Madrid en el 2003. En el otro extremo, se encuentran CCAA en las que en el curso 2018/2019 no cuentan con una propuesta de mayor grado de concreción para el alumnado con TEA, como es el caso de Cantabria y Galicia, y Castilla y León cuya iniciativa tiene carácter experimental².

Por último, en relación todavía a los años de puesta de funcionamiento, es importante señalar que en seis CCAA (Aragón, el Principado de Asturias, Castilla-La Mancha, Comunidad Valenciana, Extremadura y la Comunidad de Madrid) las iniciativas nacieron con carácter experimental unos cursos antes de consolidarse como resultado de una

${ }^{2} \mathrm{http}$ //izca.net/2018/02/22/aulas-de-comunicacion-un-proyecto-experimental-de-la-junta-que-separa-al-alumnado-condiscapacidad/ 
valoración positiva. En este sentido, como se ha comentado previamente, Castilla y León en el curso 2018/2019 se encontraban desarrollando con carácter experimental sus propuestas.

A modo de síntesis, se presenta en el cuadro 2 las distintas denominaciones y los años de aprobación de cada una de las propuestas de las distintas CCAA a través de su normativa.

Cuadro 2. Denominaciones y años de aprobación de las distintas propuestas

\begin{tabular}{|c|c|c|}
\hline CCAA & DENOMINACIÓN & PUESTA EN FUNCIONAMIENTO \\
\hline Andalucía & $\begin{array}{l}\text { Aulas } \\
\text { Específicas de } \\
\text { Educación } \\
\text { Especial } \\
\text { (pueden ser } \\
\text { especializadas } \\
\text { en TEA) }\end{array}$ & $\begin{array}{l}\text { DECRETO } 147 / 2002 \text {, de } 14 \text { de mayo, por el que se } \\
\text { establece la ordenación de la atención educativa a los } \\
\text { alumnos y alumnas con necesidades educativas especiales } \\
\text { asociadas a sus capacidades personales. } \\
\text { ORDEN de } 19 \text { de septiembre de } 2002 \text {, por la que se regula } \\
\text { la elaboración del Proyecto Curricular de los Centros } \\
\text { Específicos de Educación Especial y de la programación de } \\
\text { las aulas específicas de Educación Especial en los centros } \\
\text { ordinarios. } \\
\text { Instrucciones de } 22 \text { de junio de } 2015 \text {, de la Dirección } \\
\text { General de Participación y Equidad, por las que se } \\
\text { establece el. Protocolo de detección, identificación del } \\
\text { alumnado con necesidades específicas de apoyo educativo y } \\
\text { organización de la respuesta educativa. } \\
\text { Instrucciones de } 26 \text { de febrero de } 2018 \text {, de la } \\
\text { Viceconsejería, sobre los procedimientos de admisión y } \\
\text { matriculación del alumnado en los centros docentes } \\
\text { sostenidos con fondos públicos para el curso escolar } \\
\text { 2018/19. }\end{array}$ \\
\hline Aragón & $\begin{array}{l}\text { Centros de } \\
\text { Atención } \\
\text { Preferente a } \\
\text { alumnos con } \\
\text { TEA }\end{array}$ & $\begin{array}{l}\text { ORDEN de } 9 \text { de octubre de } 2013^{*} \text {, de la Consejera de } \\
\text { Educación, Universidad, Cultura y Deporte, por la que se } \\
\text { regulan los centros de atención preferente a alumnos con } \\
\text { trastorno del espectro autista. } \\
\text { *De forma experimental desde el curso } 2006 / 2007\end{array}$ \\
\hline $\begin{array}{l}\text { Principado de } \\
\text { Asturias }\end{array}$ & $\begin{array}{l}\text { Aulas Abiertas } \\
\text { en centros } \\
\text { ordinarios }\end{array}$ & $\begin{array}{l}\text { Instrucciones relativas a la atención del alumnado con } \\
\text { necesidades educativas especiales a través de Aulas } \\
\text { Abiertas en centros ordinarios para el curso escolar } \\
2018 / 2019^{*} \text {. } \\
\text { *De forma experimental desde el curso 2016/2017 }\end{array}$ \\
\hline Islas Baleares & $\begin{array}{l}\text { Aulas } \\
\text { Sustitutorias de } \\
\text { Centro } \\
\text { Específico }\end{array}$ & $\begin{array}{l}\text { DECRETO 39/2011, de } 29 \text { de abril, por el cual se regula } \\
\text { la atención a la diversidad y la orientación educativa en los } \\
\text { centros educativos no universitarios sostenidos con fondos } \\
\text { públicos. }\end{array}$ \\
\hline Canarias & Aulas Enclave & $\begin{array}{l}\text { DECRETO } 25 / 2018 \text {, de } 26 \text { de febrero, por el que se } \\
\text { regula la atención a la diversidad en el ámbito de las } \\
\text { enseñanzas no universitarias de la Comunidad Autónoma } \\
\text { de Canarias. } \\
\text { ORDEN de } 10 \text { de febrero de } 2016 \text {, por la que se establecen } \\
\text { las concreciones curriculares adaptadas para el alumnado } \\
\text { escolarizado en las aulas enclave y centros de educación } \\
\text { especial de la Comunidad Autónoma de Canarias. } \\
\text { ORDEN de } 13 \text { de diciembre de } 2010 \text {, por la que se regula } \\
\text { la atención al alumnado con necesidades específicas de } \\
\text { apoyo educativo en la Comunidad Autónoma de Canarias. }\end{array}$ \\
\hline $\begin{array}{l}\text { Castilla-La } \\
\text { Mancha }\end{array}$ & $\begin{array}{l}\text { Aulas abiertas } \\
\text { especializadas } \\
\text { para alumnado } \\
\text { con TEA }\end{array}$ & $\begin{array}{l}\text { ORDEN de 11/04/2014*, de la Consejería de Educación, } \\
\text { Cultura y Deportes, por la que se crean, regula y ordena el } \\
\text { funcionamiento de las Aulas Abiertas Especializadas, para } \\
\text { el alumnado con trastorno de espectro autista, en centros } \\
\text { sostenidos con fondos públicos de la Comunidad } \\
\text { Autónoma de Castilla-La Mancha. }\end{array}$ \\
\hline
\end{tabular}


*De forma experimental desde el curso 2012/2013

DECRETO 299/1997, de 25 de noviembre, sobre atención educativa al alumnado con necesidades educativas especiales.

Orientacions a l'entorn de les unitats de suport a

Unidades de Apoyo a la Educación

Cataluña Especial $(\mathrm{USEE})^{3}$

l'educació especial (USEE). Document $n^{\circ} 3$. Novembre de 2008.

Informe de valoració de les unitats de suport a l'educació especial (USEE).

Instruccions per a l'organització i el funcionament dels centres educatius públics d'educació infantil i primària I d'educació especial Curs 2008-2009.

Organització i funcionament dels centres educatius públics d'educació infantil i primària i d'educació especial Novetats (2009-2010).

* La implantación de las USEE comienza en el curso 2004/2005

\begin{tabular}{|c|c|c|}
\hline $\begin{array}{l}\text { Comunidad } \\
\text { Valenciana }\end{array}$ & $\begin{array}{l}\text { Unidades } \\
\text { Específicas de } \\
\text { Comunicación y } \\
\text { Lenguaje } \\
\text { (también } \\
\text { disponen de } \\
\text { Unidades } \\
\text { Específicas de } \\
\text { Educación } \\
\text { Especial) }\end{array}$ & $\begin{array}{l}\text { Resolución de } 27 \text { de julio de } 2016^{*} \text {, de la Dirección } \\
\text { General de Política Educativa, por la que se dictan } \\
\text { instrucciones para la organización y el funcionamiento de } \\
\text { las unidades específicas de comunicación y lenguaje } \\
\text { ubicadas en centros públicos que imparten enseñanzas de } \\
\text { Educación Infantil (2. }{ }^{\circ} \text { ciclo), Educación Primaria y } \\
\text { Educación Secundaria para el curso 2016-2017. } \\
\text { *De forma experimental desde el curso 2004/2005 }\end{array}$ \\
\hline Extremadura & $\begin{array}{l}\text { Aulas } \\
\text { especializadas } \\
\text { para alumnado } \\
\text { con TEA } \\
\text { (también } \\
\text { disponen de } \\
\text { aulas abiertas } \\
\text { especializadas de } \\
\text { Educación } \\
\text { Especial) }\end{array}$ & $\begin{array}{l}\text { ORDEN de } 12 \text { de febrero de } 2015^{*} \text { por la que se regula la } \\
\text { organización y funcionamiento de las aulas especializadas } \\
\text { para el alumnado con trastornos del espectro del } \\
\text { autismo en centros educativos sostenidos con fondos } \\
\text { públicos de la Comunidad Autónoma de Extremadura. } \\
\text { *De forma experimental previamente (no se especifica el curso } \\
\text { escolar) } \\
\text { ORDEN de } 6 \text { de julio de } 2012 \text { por la que se crean aulas } \\
\text { abiertas especializadas de Educación Especial en centros } \\
\text { ordinarios de la Comunidad Autónoma de Extremadura y } \\
\text { se regula su organización y funcionamiento. }\end{array}$ \\
\hline $\begin{array}{l}\text { Comunidad } \\
\text { de Madrid }\end{array}$ & $\begin{array}{l}\text { Centros } \\
\text { Preferentes de } \\
\text { alumnado con } \\
\text { TGD }\end{array}$ & $\begin{array}{l}\text { Documento marco para la puesta en funcionamiento de } \\
\text { escuelas infantiles y colegios de escolarización preferente } \\
\text { de alumnado con trastornos generalizados del desarrollo. } \\
9 \text { de octubre de } 2003 \text {. } \\
\text { * De forma experimental desde el curso } 2001 / 2002\end{array}$ \\
\hline $\begin{array}{l}\text { Región de } \\
\text { Murcia }\end{array}$ & $\begin{array}{l}\text { Aulas abiertas } \\
\text { especializadas } \\
\text { ( pudiendo ser } \\
\text { genéricas o } \\
\text { específicas) }\end{array}$ & $\begin{array}{l}\text { ORDEN de } 24 \text { de mayo de } 2010 \text {, de la Consejería de } \\
\text { Educación, Formación y Empleo, por la que se regulan la } \\
\text { autorización y el funcionamiento de las aulas abiertas } \\
\text { especializadas en centros ordinarios públicos y privados } \\
\text { concertados de la Comunidad Autónoma de la Región de } \\
\text { Murcia. }\end{array}$ \\
\hline $\begin{array}{l}\text { Comunidad } \\
\text { Foral de } \\
\text { Navarra }\end{array}$ & $\begin{array}{l}\text { Unidades de } \\
\text { transición TEA }\end{array}$ & $\begin{array}{l}\text { ORDEN foral 93/2008, de } 13 \text { de junio, del consejero de } \\
\text { educación por la que se regula la atención a la diversidad } \\
\text { en los centros educativos de educación infantil y primaria } \\
\text { y educación secundaria de la Comunidad Foral de } \\
\text { Navarra. }\end{array}$ \\
\hline
\end{tabular}

s Tras la aprobación del DECRETO 150/2017, de 17 de octubre, de la atención educativa al alumnado en el marco de un sistema educativo inclusivo, las USEE se transformarán en Apoyos Intensivos a la Educación Inclusiva (SIEI), sin embargo, en el curso 2018/2019 está transformación por el momento no se ha producido. 


\begin{tabular}{|c|c|c|}
\hline & & $\begin{array}{l}\text { Instrucciones para las unidades de transición / TEA. } \\
\text { Última revisión septiembre } 2017 \text {. }\end{array}$ \\
\hline País Vasco & $\begin{array}{l}\text { Aulas Estables } \\
\text { (pueden ser } \\
\text { específicamente } \\
\text { creadas para } \\
\text { TGD) }\end{array}$ & $\begin{array}{l}\text { DECRETO 118/1998, de } 23 \text { de junio, de ordenación de la } \\
\text { respuesta educativa al alumnado con necesidades } \\
\text { educativas especiales, en el marco de una escuela } \\
\text { comprensiva e integradora. } \\
\text { ORDEN de } 30 \text { de julio } 1998 \text { del Consejero de Educación, } \\
\text { Universidades e Investigación por la que se establecen } \\
\text { criterios de escolarización del alumnado con necesidades } \\
\text { educativas especiales y dotación de recursos para su } \\
\text { correcta atención en las distintas etapas del sistema } \\
\text { educativo. }\end{array}$ \\
\hline La Rioja & Aulas TEA & $\begin{array}{l}\text { Resolución de } 17 \text { de julio de } 2018 \text {, de la Dirección General } \\
\text { de Educación, por la que se dictan instrucciones para el } \\
\text { establecimiento y funcionamiento de las aulas } \\
\text { especializadas para el alumnado con necesidades } \\
\text { educativas especiales asociadas a Trastorno de Espectro } \\
\text { Autista "AULAS TEA" escolarizado en Segundo Ciclo de } \\
\text { Educación Infantil y en Educación Primaria y en } \\
\text { Educación Secundaria Obligatoria en centros sostenidos } \\
\text { con fondos públicos de la Comunidad Autónoma de La } \\
\text { Rioja. }\end{array}$ \\
\hline $\begin{array}{l}\text { Ciudades } \\
\text { Autónomas } \\
\text { de Ceuta y } \\
\text { Melilla }\end{array}$ & $\begin{array}{l}\text { Aulas Abiertas } \\
\text { Especializadas } \\
\text { para alumnado } \\
\text { con TEA }\end{array}$ & $\begin{array}{l}\text { Resolución de } 30 \text { de junio de } 2016 \text {, de la Secretaría de } \\
\text { Estado de Educación, Formación Profesional y } \\
\text { Universidades, por la que se crean, regulan y ordenan las } \\
\text { Aulas Abiertas Especializadas para el alumnado con } \\
\text { trastorno del espectro autista en centros sostenidos con } \\
\text { fondos públicos de las Ciudades Autonómicas de Ceuta y } \\
\text { Melilla. }\end{array}$ \\
\hline
\end{tabular}

Fuente: Elaboración propia.

En cuanto a la titularidad de los centros, a partir del análisis realizado podemos identificar que en todas las CCAA, a excepción del Principado de Asturias y las Ciudades Autónomas de Ceuta y Melilla, las propuestas se desarrollan en centros sostenidos con fondos públicos, es decir, centros de titularidad pública y concertada.

En el caso de la propuesta de "Aulas abiertas en centros ordinarios" en el Principado de Asturias y las "Aulas Abiertas Especializadas para alumnado con TEA" en las Ciudades Autónomas de Ceuta y Melilla, por el momento solamente se desarrollan en centros de titularidad pública, tal y como se detalla en el listado de centros que figuran en sus instrucciones.

En lo que se refiere a las etapas educativas en las que se ofertan las distintas propuestas, encontramos unanimidad de oferta en la etapa de Educación Primaria. Sin embargo, en la etapa de Educación Infantil, en la mayoría de las CCAA se ha puesto en marcha únicamente en el II ciclo y en el caso del Andalucía, Extremadura y País Vasco se indica que de forma excepcional podrán crearse sus respectivas propuestas para la etapa de Educación Infantil. Para el I ciclo, solamente se menciona de forma explícita la posibilidad de desarrollar sus propuestas en la Comunidad de Madrid4 y la Región de Murcia.

Respecto a la etapa de Educación Secundaria Obligatoria, todas las CCAA ofertan sus iniciativas para esta etapa a excepción del Principado de Asturias y las Ciudades

${ }^{4}$ En el caso de Madrid su Documento Marco únicamente hace referencia a Escuelas Infantiles y colegios, sin embargo, a partir del curso 2003/2004 su propuesta se extiende a la Educación Secundaria Obligatoria. 
Autónomas de Ceuta y Melilla cuya iniciativa se circunscribe únicamente al II ciclo de Educación Infantil y a Educación Primaria, previsiblemente por haber sido implementadas recientemente. En el caso de la Comunidad Foral de Navarra se establece que después de la escolarización del alumnado con TEA en las "Unidades de Transición" en la etapa de Educación Primaria podrá incorporarse a una "Unidad de Currículo Específico", entendida como "una estructura flexible creada para facilitar la escolarización en centros ordinarios de Secundaria Obligatoria a alumnado con necesidades educativas especiales asociada a discapacidad psíquica leve o moderada, que ha estado integrado en la etapa de Educación Primaria, o a un centro de educación especial".

Por último, después de la etapa de Educación Secundaria Obligatoria únicamente el País Vasco establece, con el mismo carácter de las "Aulas Estables", la posibilidad de que el alumnado que proviene de estas aulas pueda tener continuidad a través de las "Aulas de Aprendizaje de Tareas". Estas aulas de aprendizaje de tareas tienen el objetivo de "fomentar la preparación laboral y para la vida activa de los alumnos y alumnas con necesidades educativas especiales ligadas a un retraso mental. La característica fundamental de estas aulas es la de servir de transición del periodo educativo a la vida adulta y al mundo del trabajo".

En cuanto a la dimensión relacionada con el encuadre conceptual de cada una de las iniciativas, en relación a la modalidad de escolarización en la que se encuadra cada una de las propuestas, es importante advertir dos cuestiones. En primer lugar, todas las CCAA, sin excepción, sitúan sus propuestas en el marco de un enfoque inclusivo bajo los principios de normalización e inclusión, a excepción del País Vasco que, como consecuencia del año de su normativa, hace referencia a que su iniciativa se enmarca en una escuela comprensiva e integradora. Es decir, que podríamos interpretar que todas las CCAA entienden sus propuestas como herramientas para avanzar hacia un sistema educativo inclusivo.

Sin embargo, la segunda cuestión a tener en cuenta hace referencia a la modalidad de escolarización en la que se enmarcan las propuestas de las distintas CCAA. En este sentido, se ha encontrado una referencia explícita a que la modalidad de escolarización es ordinaria con apoyos especializados en el caso de Aragón, Comunidad Valenciana, Comunidad de Madrid, Comunidad Foral de Navarra y la Rioja.

Por otra parte, las CCAA de El Principado de Asturias, Castilla-La Mancha, y las Ciudades Autonómicas de Ceuta y Melilla no hacen una referencia explícita a la modalidad de escolarización en la que se encuadra su propuesta, por lo que se entiende que, si no hay una referencia explícita que indique lo contrario, en estas CCAA las respectivas propuestas también se enmarcan dentro de la modalidad ordinaria con apoyos especializados.

En el caso de Andalucía, Canarias y el País Vasco, otorgan a sus propuestas identidad propia como una modalidad concreta diferente a las tradicionales, De esta forma son denominadas: Modalidad A: aula de educación especial en centro ordinario en Andalucía, Aula Enclave en el caso de Canarias y Aulas Estables en centro ordinarios en el País Vasco.

Por último, encontramos, por el contrario, cuatro CCAA que enmarcan sus propuestas en una modalidad combinada o de educación especial. En el caso las Islas Baleares, las "Aulas sustitutorias en centros específicos" se constituyen como una opción dentro de la modalidad de educación especial para determinado alumno escolarizado en el segundo ciclo de Educación Infantil, Educación Primaria y Educación Secundaria Obligatoria, pudiendo desarrollarse también programas de Transición a la Vida Adulta. 
En Cataluña, en las "Unidades de Apoyo a la Educación Especial" se hace referencia a que sus destinatarios son "alumnos con NEE graves y permanentes que serían susceptibles de ser escolarizados en centros específicos. Este perfil de alumnado corresponde a lo que habitualmente reconocemos como de Centro de Educación Especial cuando estas necesidades educativas sólo podían ser atendidas en esta modalidad de escolarización".

Por su parte, Extremadura enmarca su propuesta en una modalidad combinada señalando ando que "la modalidad de escolarización combinada entre centro ordinario y aula especializada se entiende en estas aulas como escolarización en centro ordinario con adaptaciones curriculares significativas y/o de acceso, utilizando el aula especializada como recurso"

Por último, la Región de Murcia señala que la escolarización se realiza a través de una Comisión específica de Educación especial, por lo que en caso de no disponer de plazas en la modalidad que ofertan, el alumnado, de forma transitoria, podría escolarizarse en un Centro de Educación Especial.

Respecto al perfil del alumnado destinatario, podemos clasificar a las CCAA en tres grandes grupos:

- CCAA en las que sus propuestas se vinculan únicamente al alumnado con TEA, TGD o con indicadores compatibles: es el caso de Aragón, Castilla-La Mancha, Extremadura, la Comunidad de Madrid, La Rioja, y las Ciudades Autónomas de Ceuta y Melilla.

- CCAA en las que sus propuestas además del alumnado con TEA o TGD, abren el abanico a otras dificultades relacionadas con la comunicación: es el caso del Principado de Asturias y la Comunidad Valenciana (en el caso de las unidades específicas de comunicación y lenguaje).

- CCAA en las que sus propuestas engloban varias necesidades educativas especiales, es el caso de Andalucía, Islas Baleares, Canarias, Cataluña, Comunidad Valenciana (en el caso de las Unidades Específicas de Educación Especial), Extremadura, la Región de Murcia, la Comunidad Foral de Navarra y el País Vasco.

Por último, a pesar de que las normas identificadas en Andalucía, Extremadura, la Región de Murcia y El País Vasco responden a distintos colectivos de alumnado con necesidades educativas especiales, se ha podido constatar la posibilidad de especialización de sus propuestas para el alumnado con TEA, es decir que sus propuestas generales se desarrollen en algunos casos exclusivamente para alumnado con TEA.

En cuanto a la ratio que cada CCAA delimita para sus respectivas propuestas aparecen grandes diferencias en dos niveles. El primer nivel hace referencia a las distintas ratios que establece cada CCAA en función de las etapas educativas en la que se oferta. El segundo nivel se vincula con las diferencias existentes entre cada CCAA cuando se comparan las ratios que cada una de ellas delimita, siendo imprescindible tener en cuenta, como se ha comentario en el punto anterior, que el perfil del alumnado destinatario claramente es diferente entre unas y otras.

A continuación, se presenta el cuadro 3 en la que se especifican las ratios en cada CCAA en función de las etapas educativas.

Cuadro 3. Ratios establecidas en función de las distintas etapas educativas en cada CCAA. 


\begin{tabular}{|c|c|c|c|c|}
\hline CCAA & $\begin{array}{c}\text { EDUCACIÓN } \\
\text { INFANTIL } \\
\left(1^{\circ} \text { CICLO }\right) \\
\end{array}$ & $\begin{array}{l}\text { EDUCACIÓN } \\
\text { INFANTIL } \\
\left(2^{\circ} \text { CICLO }\right) \\
\end{array}$ & $\begin{array}{l}\text { EDUCACIÓN } \\
\text { PRIMARIA }\end{array}$ & $\begin{array}{c}\text { EDUCACIÓN } \\
\text { SECUNDARIA } \\
\text { OBLIGATORIA } \\
\end{array}$ \\
\hline Andalucía & - & $\begin{array}{l}\text { Se establecen las } \\
\text { siguientes ratios } \\
\text { en función de los } \\
\text { diagnósticos que } \\
\text { se detallan a } \\
\text { continuación: } \\
\text {-Psíquicos: 6-8. } \\
\text {-Sensoriales: 6-8. } \\
\text { Físicos/Motóricos: } \\
\text { 8-10. } \\
\text {-Autistas o } \\
\text { Psicóticos: 3-5 } \\
\text {-Plurideficientes: } \\
\text { 4-6. } \\
\text {-Unidades que } \\
\text { escolarizan } \\
\text { alumnado de } \\
\text { diferentes } \\
\text { discapacidades: } 5\end{array}$ & $\begin{array}{l}\text { Se establecen las } \\
\text { siguientes ratios } \\
\text { en función de los } \\
\text { diagnósticos que } \\
\text { se detallan a } \\
\text { continuación: } \\
\text {-Psíquicos: 6-8. } \\
\text {-Sensoriales: 6-8. } \\
\text { Físicos/Motóricos: } \\
\text { 8-10. } \\
\text {-Autistas o } \\
\text { Psicóticos: 3-5 } \\
\text {-Plurideficientes: } \\
\text { 4-6. } \\
\text {-Unidades que } \\
\text { escolarizan } \\
\text { alumnado de } \\
\text { diferentes } \\
\text { discapacidades: } 5\end{array}$ & $\begin{array}{l}\text { Se establecen las } \\
\text { siguientes ratios } \\
\text { en función de los } \\
\text { diagnósticos que } \\
\text { se detallan a } \\
\text { continuación: } \\
\text {-Psíquicos: 6-8. } \\
\text {-Sensoriales: 6-8. } \\
\text { Físicos/Motóricos: } \\
\text { 8-10. } \\
\text {-Autistas o } \\
\text { Psicóticos: } 3-5 \\
\text {-Plurideficientes: } \\
\text { 4-6. } \\
\text {-Unidades que } \\
\text { escolarizan } \\
\text { alumnado de } \\
\text { diferentes } \\
\text { discapacidades: } 5 \\
\text {-Programas de } \\
\text { Transición a la } \\
\text { Vida Adulta y } \\
\text { Laboral: } 8\end{array}$ \\
\hline Aragón & - & $\begin{array}{l}\text { Al margen de las } \\
\text { líneas de los } \\
\text { centros, se } \\
\text { reservan una o } \\
\text { dos líneas por } \\
\text { centros para esta } \\
\text { modalidad. } \\
\text { Se reservan } 7 \\
\text { plazas en centros } \\
\text { con reserva de } \\
\text { una línea y } 14 \\
\text { plazas en centros } \\
\text { con reserva de } 2 \\
\text { líneas. } \\
\text { Las vacantes } \\
\text { reservadas que } \\
\text { no se cubran se } \\
\text { reservan. }\end{array}$ & $\begin{array}{l}\text { Al margen de las } \\
\text { líneas de los } \\
\text { centros, se } \\
\text { reservan una o } \\
\text { dos líneas por } \\
\text { centros para esta } \\
\text { modalidad. } \\
\text { Se reservan } 7 \\
\text { plazas en centros } \\
\text { con reserva de } \\
\text { una línea y } 14 \\
\text { plazas en centros } \\
\text { con reserva de } 2 \\
\text { líneas. } \\
\text { Las vacantes } \\
\text { reservadas que } \\
\text { no se cubran se } \\
\text { reservan. }\end{array}$ & $\begin{array}{l}\text { Se reservan un } \\
\text { máximo de dos } \\
\text { máximos en cada } \\
\text { centro, } \\
\text { pudiéndose ser } \\
\text { ocupadas las } \\
\text { plazas por un } \\
\text { máximo de } 8 \\
\text { alumnos/as } \\
\text { Las vacantes que } \\
\text { no se cubran no } \\
\text { se reservan }\end{array}$ \\
\hline $\begin{array}{l}\text { Principado } \\
\text { de Asturias }\end{array}$ & - & $\begin{array}{l}\text { Mínimo } 3 \\
\text { alumnos/as y } \\
\text { máximo } 5\end{array}$ & $\begin{array}{l}\text { Mínimo } 3 \\
\text { alumnos/as y } \\
\text { máximo } 5\end{array}$ & - \\
\hline $\begin{array}{l}\text { Islas } \\
\text { Baleares }\end{array}$ & - & $\begin{array}{l}\text { Máximo } 7 \\
\text { alumnos, pero el } \\
\text { número puede } \\
\text { ser inferior su } \\
\text { predominan en } \\
\text { ella alumnado } \\
\text { con discapacidad } \\
\text { motriz graves, } \\
\text { con autismo o } \\
\text { con trastorno de } \\
\text { la personalidad. }\end{array}$ & $\begin{array}{l}\text { Máximo } 7 \\
\text { alumnos, pero el } \\
\text { número puede } \\
\text { ser inferior su } \\
\text { predominan en } \\
\text { ella alumnado } \\
\text { con discapacidad } \\
\text { motriz graves, } \\
\text { con autismo o } \\
\text { con trastorno de } \\
\text { la personalidad. }\end{array}$ & $\begin{array}{l}\text { Máximo } 7 \\
\text { alumnos, pero el } \\
\text { número puede } \\
\text { ser inferior su } \\
\text { predominan en } \\
\text { ella alumnado } \\
\text { con discapacidad } \\
\text { motriz graves, } \\
\text { con autismo o } \\
\text { con trastorno de } \\
\text { la personalidad. }\end{array}$ \\
\hline
\end{tabular}




\begin{tabular}{|c|c|c|c|c|}
\hline Canarias & - & $\begin{array}{l}\text { Mínimo } 3 \text { y } \\
\text { máximo } 5 \\
\text { alumnos/as }\end{array}$ & $\begin{array}{l}\text { Mínimo } 3 \text { y } \\
\text { máximo } 5 \\
\text { alumnos/as }\end{array}$ & $\begin{array}{l}\text { Mínimo tres y } \\
\text { máximo } 6 \\
\text { alumnos/as en } \\
\text { IES }\end{array}$ \\
\hline $\begin{array}{l}\text { Castilla-La } \\
\text { Mancha }\end{array}$ & - & $\begin{array}{l}\text { Entre } 4 \text { y } 6 \\
\text { alumnos/as }\end{array}$ & $\begin{array}{l}\text { Entre } 4 \text { y } 6 \\
\text { alumnos/as }\end{array}$ & $\begin{array}{l}\text { Entre } 4 \text { y } 7 \\
\text { alumnos/as }\end{array}$ \\
\hline Cataluña & - & $\begin{array}{c}\text { Entre } 5 \text { y } 8 \\
\text { alumnos/as }\end{array}$ & $\begin{array}{c}\text { Entre } 5 \text { y } 8 \\
\text { alumnos/as }\end{array}$ & $\begin{array}{c}\text { Entre } 4 \text { y } 8 \\
\text { alumnos/as }\end{array}$ \\
\hline Extremadura & - & $\begin{array}{l}\text { Entre } 3 \text { y } 6 \\
\text { alumnos/as }\end{array}$ & $\begin{array}{l}\text { Entre } 3 \text { y } 6 \\
\text { alumnos/as }\end{array}$ & $\begin{array}{l}\text { Entre } 3 \text { y } 6 \\
\text { alumnos/as }\end{array}$ \\
\hline La Rioja & - & $\begin{array}{l}\text { Máximo } 8 \\
\text { alumnos/ as } \\
\text { pudiéndose } \\
\text { incrementar este } \\
\text { número hasta un } \\
10 \% \text {. }\end{array}$ & $\begin{array}{l}\text { Máximo } 8 \\
\text { alumnos/ as } \\
\text { pudiéndose } \\
\text { incrementar este } \\
\text { número hasta un } \\
10 \% \text {. }\end{array}$ & $\begin{array}{l}\text { Máximo } 8 \\
\text { alumnos/ as } \\
\text { pudiéndose } \\
\text { incrementar este } \\
\text { número hasta un } \\
10 \% \text {. }\end{array}$ \\
\hline $\begin{array}{l}\text { Comunidad } \\
\text { de Madrid }\end{array}$ & $\begin{array}{l}\text { Hasta un } \\
\text { máximo de } \\
5 \text { a } \\
\text { alumnos/as }\end{array}$ & $\begin{array}{l}\text { Hasta un máximo } \\
\text { de } 5 \text { a } \\
\text { alumnos/as. }\end{array}$ & $\begin{array}{l}\text { Hasta un máximo } \\
\text { de } 5 \text { a } \\
\text { alumnos/as. }\end{array}$ & $\begin{array}{l}\text { Hasta un máximo } \\
\text { de } 5 \text { a } \\
\text { alumnos/as. }\end{array}$ \\
\hline $\begin{array}{l}\text { Región de } \\
\text { Murcia }\end{array}$ & $\begin{array}{l}\text { Máximo } 6 \\
\text { alumnos/as }\end{array}$ & $\begin{array}{l}\text { Entre } 3 \text { y } 5 \\
\text { alumnos/as }\end{array}$ & $\begin{array}{l}\text { Entre } 4 \text { y } 6 \\
\text { alumnos/as. }\end{array}$ & $\begin{array}{l}\text { Entre } 4 \text { y } 6 \\
\text { alumnos/as. }\end{array}$ \\
\hline $\begin{array}{l}\text { Comunidad } \\
\text { Foral de } \\
\text { Navarra }\end{array}$ & - & $\begin{array}{l}\text { Entre } 3 \text { y } 5 \\
\text { alumnos/as. }\end{array}$ & $\begin{array}{l}\text { Entre } 3 \text { y } 5 \\
\text { alumnos/as. }\end{array}$ & - \\
\hline País Vasco & - & $\begin{array}{l}\text { Hasta un máximo } \\
\text { de } 5 \text { a } \\
\text { alumnos/as. }\end{array}$ & $\begin{array}{l}\text { Hasta un máximo } \\
\text { de } 5 \text { a } \\
\text { alumnos/as. }\end{array}$ & $\begin{array}{l}\text { Hasta un máximo } \\
\text { de } 5 \text { a } \\
\text { alumnos/as. }\end{array}$ \\
\hline $\begin{array}{l}\text { Comunidad } \\
\text { Valenciana }\end{array}$ & - & $\begin{array}{l}\text { Entre } 3 \text { y } 5 \\
\text { alumnos/as de la } \\
\text { 5modalidad A y } \\
\text { hasta llegar a } 8 \\
\text { alumnos/as de la } \\
\text { modalidad B. }\end{array}$ & $\begin{array}{l}\text { Entre } 3 \text { y } 5 \\
\text { alumnos/as de la } \\
\text { modalidad A y } \\
\text { hasta llegar a } 8 \\
\text { alumnos/as de la } \\
\text { modalidad B. }\end{array}$ & $\begin{array}{l}\text { Entre } 3 \text { y } 5 \\
\text { alumnos/as de la } \\
\text { modalidad A y } \\
\text { hasta llegar a } 8 \\
\text { alumnos/as de la } \\
\text { modalidad B. }\end{array}$ \\
\hline $\begin{array}{l}\text { Ciudades } \\
\text { Autonómicas } \\
\text { de Ceuta y } \\
\text { Melilla }\end{array}$ & - & $\begin{array}{l}\text { Máximo } 6 \\
\text { alumnos/ as }\end{array}$ & $\begin{array}{l}\text { Máximo } 6 \\
\text { alumnos/ as. }\end{array}$ & - \\
\hline
\end{tabular}

Nota: "-" hace referncia a que no se desarrolla la propuesta en esta etapa.

Fuente: Elaboración propia.

Por último, únicamente en la normativa de Cataluña y la Región de Murcia se establece un número máximo de "Unidades de Apoyo a la Educación Especial" y "Aulas abiertas especializadas", respectivamente, en los centros educativos. Específicamente Cataluña delimita un número máximo de tres unidades por centro y la Región de Murcia dos unidades en los centros públicos de Educación Infantil, Educación Primaria y Educación

\footnotetext{
${ }^{5}$ La Modalidad A hace referencia al alumnado que requiere más del 40\% del horario semanal estar en la unidad específica mientras que la Modalidad B el tiempo que requiere es inferior a este porcentaje. También se identifica la posibilidad de atender a un mayor número de alumnado en función de necesidades sobrevenidas.
} 
Secundaria y tres en los centros privados concertados donde se impartan todas las etapas educativas.

\section{Discusión y conclusiones}

$\mathrm{El}$ análisis presentado permite documentar las distintas posibilidades educativas que las administraciones autonómicas impulsan para la escolarización del alumnado con TEA. Como se ha comentado anteriormente, es importante destacar que estos datos tienen algunas limitaciones principalmente al tratarse de un análisis exclusivamente normativo a través de la legislación existente en cada CCAA, y haberse presentado únicamente el análisis de dos dimensiones. A pesar de ello, creemos que el trabajo desarrollado es relevante teniendo en cuenta el escaso número de investigaciones relacionadas con la temática en nuestro país.

De hecho, los datos recogidos permiten extraer algunas conclusiones interesantes. En primer lugar, podemos destacar que, en la actualidad, catorce CCAA y las Ciudades Autónomas de Ceuta y Melilla ofertan propuestas educativas que permiten al alumnado con TEA estar escolarizados en centros ordinarios combinando una intervención educativa y el trabajo con apoyos intensivos junto la posibilidad de convivir con sus iguales en un entorno educativo ordinario. Este dato sin duda es positivo, ya que se podría interpretar esta situación como un avance en el proceso de inclusión educativa, al menos para este colectivo de alumnado en nuestro sistema.

Sin embargo, el análisis presentado permite contrastar que este avance no se ha desarrollado de forma homogénea a lo largo de todo el Estado y que en el planteamiento de algunas de las propuestas se puede constatar una falta de comprensión de lo que debería suponer el principio de inclusión educativa, principio que se regula en la actual la Ley de Educación. Parecería que la persistencia en el debate entre integración versus inclusión no ayuda a centrar la discusión en si los alumnos deben adaptarse al sistema o si por el contrario es el sistema el que debe adaptarse a ellos (Echeita y Verdugo, 2004). A modo de ejemplo, esta situación se aprecia claramente en la estrategia adoptada en la dotación del profesorado de apoyo especializado a los centros. En lugar de que todos los centros educativos cuenten con al menos un mínimo de profesorado de apoyo para atender a la diversidad del alumnado, este profesorado continúa adscribiéndose en función del número de alumnado y el tipo de necesidades que presentan.

Una de las mayores desigualdades se aprecia en las distintas velocidades, es decir en el ritmo en el que las CCAA han ido incorporando propuestas inclusivas en la atención al alumnado con TEA. En un extremo, se situaría el País Vasco, CCAA que en el "siglo pasado" ya apostó por las Aulas Estables como fórmula integradora para el alumnado con mayores necesidades educativas, y en el otro extremo CCAA como Cantabria, Castilla y León, y Galicia, que por el momento no cuenta con una propuesta inclusiva para el alumnado con TEA ni para otro colectivo. Entre medias, se distribuyen las CCAA que de forma progresiva han ido avanzando a lo largo de estos veinte años que separan la propuesta pionera de las más recientes.

En este sentido, es importante destacar el carácter experimental de las propuestas expuestas en sus inicios. Se ha podido constatar que, al menos en seis CCAA, las iniciativas nacieron de forma experimental con un pequeño grupo de alumnado para posteriormente. Esta circunstancia podría interpretarse como un indicador de la complejidad que requiere 
impulsar un proceso de inclusión educativa para el alumnado con mayores dificultades, por lo que resulta imprescindible realizar un seguimiento sistemático y evaluación de este proceso para reajustar cuantos aspectos sean necesarios. No obstante, también es importante que, tras este análisis, las propuestas vayan consolidándose y generalizando lo aprendido de forma progresiva a otros centros.

Respecto al nombre seleccionado para denominar las propuestas, la mayoría de las CCAA han apostado por la denominación de "aula" o "unidades", únicamente dos CCAA, Aragón y la Comunidad de Madrid se han decantado por la denominación de "centros". Sin embargo, cabría entender que estas aulas, dado que se constituyen bajo un paradigma inclusivo, suponen un espacio abierto de intervención especializado, en palabras del Decreto 104/2018 por el que se desarrollan los principios de equidad y de inclusión en el sistema educativo valenciano "un recurso integrado en el centro del que forma parte, que contribuye a la transformación de este en un centro inclusivo de referencia para la atención al alumnado con necesidades educativas especiales".

Este planteamiento sitúa al centro educativo como el eje vertebrador de la respuesta a la inclusión y por tanto hace partícipe a todas sus estructuras y profesionales del proceso de inclusión del alumnado. Durán y otros (2005) a través de la utilización de la herramienta Index for Inclusion en centros de Cataluña, la Comunidad de Madrid, y el País Vasco ya apuntaron la relevancia de esta cuestión al concluir, entre otros aspectos, que avanzar hacia fórmulas inclusivas supone un proceso de complejo y singular en el que la participación de toda la comunidad educativa, además de ser una garantía de éxito, también enriquece y facilita la incorporación de las voluntades de los distintos actores implicados.

Otra de las cuestiones analizadas se relaciona con la titularidad de los centros en los que se ofertan las propuestas. En los datos recogidos se puede constatar que todas las CCAA, a excepción del Principado de Asturias y las Ciudades Autónomas de Ceuta y Melilla, ofrecen sus propuestas a todos los centros sostenidos con fondos públicos lo que se puede interpretar como un dato positivo al mantener el mismo nivel de exigencia a las dos redes. Sin embargo, en futuros trabajos sería interesante analizar con detalle la distribución del alumnado entre ellas para comprobar si existe un equilibrio entre ambas.

En relación a las etapas educativas en las que se ofrecen las distintas propuestas, como mostraba la tabla 3 se puede apreciar una desigualdad respecto a las etapas educativas y un escaso cuidado a las etapas no obligatorias, y más en concreto al primer ciclo de Educación Infantil pero especialmente a etapa postobligatoria.

Resulta complejo entender los motivos por el que son escasas las propuestas en el primer ciclo de Educación Infantil, especialmente cuando existe gran unanimidad en las repercusiones positivas de la intervención temprana (Fuentes et al., 2006). Tal vez, la frecuencia de las intervenciones externas en otros servicios no educativos, la no obligatoriedad de la Educación Infantil y la falta de integración de ambos ciclos educativos de esta primera etapa en los mismos centros educativos, puedan estar entre alguno de los motivos.

Por su parte, en el caso de las etapas postobligatorias, solamente el País Vasco, una vez más de forma pionera, apuesta por dar continuidad a sus Aulas Estables con las Aulas de Aprendizaje de Tareas. Este dato llama la atención cuando distintos trabajos apuntan a la 
necesidad de pensar en cómo avanzar hacia una educación más inclusiva en las etapas postobligatorias dado sus escasos avances (Echeita y Verdugo, 2004).

Para terminar, dos últimas reflexiones que también ejemplifican que todavía hay que avanzar en la comprensión de lo que el proceso de inclusión debería suponer y muestran la desigualdad entre las CCAA. Por una parte, se puede apreciar el mantenimiento de las distintas modalidades de escolarización, entendidas como diferentes fórmulas de escolarización, pero con diferencias notables en cuanto a la tipología entre las distintas propuestas lo que remite a la necesidad de una mayor homogeneidad entre las distintas CCAA.

Por último, y a pesar de que el análisis presentado ha versado sobre un colectivo muy concreto de alumnado se han podido constatar notables diferencias como consecuencia de la distinta regulación de las diferentes administraciones, lo que supone que un mismo alumno o alumna podría tener una respuesta más o menos inclusiva en función de las CCAA en la que resida.

Además de esta circunstancia, incluso dentro del alumnado con TEA, la existencia de diferentes perfiles para cada una de las propuestas expuestas implica que un alumno con TEA podría ser un candidato a beneficiarse de una propuesta inclusiva en una CCAA, pero no en otra que también contase con una iniciativa de características similares al no cumplir con el perfil destinatario.

Todas estas circunstancias hacen sensato pensar en la urgencia de trabajar desde la Administración central de forma conjunta con las distintas Comunidades Autónomas en relación a temas de interés común que ayuden, de una forma contextualizada, a resolver algunas dificultades compartidas (Martínez, 2010). Para ello, el desarrollo de una norma común en materia de inclusión educativa por parte de la Administración central, previsiblemente ayudaría a garantizar la equidad de nuestro sistema y a que las CCAA debieran alinearse a ella sin que esta herramienta limitase sus legítimas competencias.

En este sentido, y a pesar del largo camino que debe recorrer nuestro sistema educativo para hacer efectivo el derecho a una educación inclusiva de todo el alumnado, cabe señalar la posibilidad que parece ofrecer el nuevo proyecto de Ley Aprobado por el Consejo de Ministros el pasado 15 de febrero de $2019^{6}$, y que como consecuencia de la inestabilidad política se encuentra paralizado y a la espera de su trámite parlamentario.

Este proyecto de Ley es el primero que incorpora explícitamente y se alinea con la Convención de los Derechos de las Personas con Discapacidad de Naciones Unidas. Además, en la Disposición adicional cuarta se alude a la necesidad de evolucionar hacia la escolarización del alumnado con necesidades educativas especiales a contextos ordinarios a través de un plan articulado con las CCAA para que, en el plazo de diez años, los centros ordinarios cuenten con las garantías suficientes para poder atender en las mejores condiciones posibles al alumnado con discapacidad. Y por último, incorpora un paquete de medidas que recuperan la comprensividad del sistema educativo. Sin duda alguna, estos tres aspectos arrojan esperanzas a retomar el compromiso como país para liderar un proceso profundo de transformación educativa que garantice la presencia, participación y aprendizaje de todos y cada uno de nuestros estudiantes.

${ }^{6}$ http://www.educacionyfp.gob.es/prensa/actualidad/2019/02/20190215-proyectoley.html 


\section{Referencias}

Bergenson, T., Heuschel, M., Harmon, R., Gille, D. y Colwell, M. (2003). Los aspectos pedagógicos de los trastornos del espectro autista. Office of Superintendent of Public Instruction.

Charman, T., Pellicano, L., Peacey, L., Peacey, N., Forward, K. y Dockrell, J. (2011). What is good practice in autism education? Institute of Education.

Durán, D., Echeita, G., Giné, C., Miguel, E., Ruiz, C. y Sandoval, M. (2005). Primeras experiencias de uso de la Guía para la evaluación y mejora de la educación inclusiva (Index for Inclusion) en el estado Español. REICE. Revista Iberoamericana sobre Calidad, Eficacia y Cambio en Educación, 3(1), 464-467.

Echeita, G. (2006). Educación para la inclusión o educación sin exclusiones. Narcea.

Echeita, G. y Verdugo, M. (Eds.). (2004): La declaración de salamanca sobre necesidades educativas especiales diez años después. Valoración y prospectiva. Publicaciones INICO.

Fuentes, J., Ferrari, M. J., Boada, L., Touriño, E., Artigas, J., Belinchón, M., Muñoz, J. A., Hervás, A., Canal, R., Hernández, J. M., Díez-Cuervo, A., Idiazábal, M. A., Mulas, F., Palacios, S., Tamarit, J., Martos, J. y Posada, M. (2006). Guía de buena práctica para el tratamiento de los trastornos del espectro autista. Revista Española de Neurología, 43, 425-438. https://doi.org/ 10.33588/rn.4307.2005750

Kanner, L. (1943). Autistic disturbances of affective contact. Nervous Child, 2, 217-250.

Marchesi, A. (2002). La práctica de las escuelas inclusivas. En A. Marchesi, C. Coll y J. Palacios, J. (Coord.) Desarrollo psicológico y educación. Trastornos del desarrollo y necesidades educativas especiales. Alianza Editorial.

Martínez, B. (2010). Luces y sombras de las medidas de atención a la diversidad en el camino de la inclusión educativa. Revista Interuniversitaria de Formación del Profesorado, 70, 165-184.

Martínez, R., De Haro, R. y Escarbajal, A (2010). Una aproximación a la educación inclusiva en España. Revista de Educación Inclusiva, 3(1), 149-164.

Martins, M., Harris, S. y Handleman, J. (2014). Supporting inclusive education. En G. Volkmar, S.J. Rogers, R. Paul y K.A Pelpherey (Eds.), Handbook of autism and pervasive developmental disorders. Assesment, intervetion, and policy (pp. 813-825). John Wiley and Sons.

Palomo, R. (2017). Los alumnos con trastorno del espectro del autismo. En A. Marchesi, C. Coll y J. Palacios, J. (Coords.), Desarrollo psicológico y educación. Respuestas educativas a las dificultades de aprendizaje y desarrollo (pp. 445-485). Madrid. Alianza Editorial.

Rivière, A. (2001). Autismo. Orientaciones para la intervención educativa. Madrid: Editorial Trotta.

Saleh, L. (2004). De Torremolinos a Salamanca y más allá. Un homenaje a España. En G. Echeita y M. Verdugo, La Declaración de Salamanca sobre necesidades educativas especiales diez años después. Valoración y prospectiva (pp. 23-34). Publicaciones INICO.

Sandoval, M., Simón, C. y Echeita, G. (2012). Análisis y valoración crítica de las funciones del profesorado de apoyo desde la educación inclusiva. Revista de Educación, $N^{o}$ Extraordinario, $117-137$.

Strain, P. y Bovey, E. (2011). Randomized, controlled trial of the LEAP model of early intervention for young children with autism spectrum disorders. Topics in Early Childhood Special Education, 31(3), 133-154. https://doi.org/10.1177/0271121411408740 
Verdugo, M., Amor, A., Fernández, M., Navas, P. y Calvo, I. (2018). La regulación de la inclusión educativa del alumnado con discapacidad intelectual: Una reforma pendiente. Siglo Cero. 49(2), 27-58. https://doi.org/10.14201/scero20184922758

Vislie, L. (1995). Integration policies, scholl reforms and the organization of schooling for handicapped pupils in western societies. En C. Clark, A. Dyson y A. Millward (Eds.), Toward inclusive schools? (pp. 42-53). David Fulton.

\section{Breve CV de las autoras}

\section{Belén de la Torre González}

Licenciada en Psicología por la Universidad Complutense de Madrid (UCM), en Psicopedagogía por la Universidad Nacional a Distancia (UNED) y Máster en Intervención Educativa en Contextos Sociales por la UNED. En la actualidad cursando estudios de Doctorado en la Facultad de Psicología de la Universidad Autónoma de Madrid (UAM). Durante años, técnico de la Organización de Estados Iberoamericanos para la Educación, la Ciencia y la Cultura (OEI) como responsable de los programas de cooperación en materia de calidad educativa, formación docente y educación en valores. Desde el 2016, orientadora educativa de la Comunidad de Madrid y asesora Técnico docente (curso 2018-19) del área de Inclusión Educativa del Centro Nacional de Innovación e Investigación Educativa (CNIIE), del Ministerio de Educación y Formación Profesional. En la actualidad, orientadora del EOEP de Móstoles. ORCID ID: https://orcid.org/o000-0002-7196-614X. Email: belendelatorre22@hotmail

\section{Elena Martín}

Catedrática de Psicología Evolutiva y de la Educación en la Universidad Autónoma de Madrid. Coordinadora del Máster Oficial en Psicología de la Educación de la UAM. Subdirectora y Directora General del Ministerio de Educación y Ciencia, cargos desempeñados durante 11 años (1985-96) formando parte del grupo responsable de la preparación y puesta en marcha de la Reforma Educativa de la LOGSE. Miembro del Instituto de Evaluación y Asesoramiento de centros Docentes (IDEA) que lleva a cabo evaluaciones de programas y de centros escolares. Sus publicaciones e investigaciones se centran en los temas relacionados con las concepciones de profesores y estudiantes; la lectura y la escritura en los procesos de aprendizaje; el currículum; el asesoramiento psicopedagógico; y la evaluación educativa desde la perspectiva del aprendizaje, de los centros y del sistema. ORCID ID: https://orcid.org/o000-0003-3445-023X. Email: elena.martin@uam.es 